\title{
Evaluation of Seismic Image Quality - part II: Assessment of Velocity Models
}

\author{
A.J. Berkhout*, J. Thorbecke \\ Delft University of Technology, Laboratory of Seismics and Acoustics, \\ P. 0. Box 5046, 2600 GA Delft, The Netherlands
}

\section{SUMMARY}

Areal shot records due to focused sources (CFP gathers) are used to generate image gathers. The high quality of those image gathers allow an accurate assessment of the underlying velocity model.

\section{Introduction}

If we assume that the correct migration algorithm has been used (see part I), errors in seismic images may still be present due to errors in the underlying velocity model. The standard way of detecting velocity errors is to search for misalignments in image gathers. However, the low signal-to-noise ratio of image gathers may make the analysis difficult. Image gathers from CFP gathers have the significant advantage that each member trace involves the contribution of many Fresnel zones and therefore has a relatively high signal content.

\section{The forward model}

The so-called WRW-model is an attractive starting point for the derivation of seismic processing algorithms. The discrete version for primary reflections can be formulated as (Berkhout, 1984):

$$
\begin{aligned}
& \vec{P}^{-}\left(z_{o}\right)=\mathbf{X}\left(z_{o}, z_{o}\right) \vec{S}\left(z_{o}\right) \\
& \vec{P}\left(z_{o}\right)=\mathbf{D}\left(z_{o}\right) \mathbf{X}\left(z_{o}, z_{o}\right) \vec{S}\left(z_{o}\right) \\
& \mathbf{P}\left(z_{o}\right)=\mathbf{D}\left(z_{o}\right) \mathbf{X}\left(z_{o}, z_{o}\right) \mathbf{S}\left(z_{o}\right)
\end{aligned}
$$

where for primary reflections (Figure la):

$$
\mathbf{X}\left(z_{o}, z_{o}\right)=\sum_{m=1}^{\infty} \mathbf{W}\left(z_{o}, z_{m}\right) \mathbf{R}^{+}\left(z_{m}\right) \mathbf{W}\left(z_{m}, z_{o}\right)=\mathbf{W}_{o}^{-} \mathbf{R}^{+} \mathbf{W}_{o}
$$

In the above equations wavefield vector $\vec{P}^{-}\left(z_{o}\right)$ equals the upgoing wavefield at the surface $\left(z=z_{o}\right)$, data vector $\vec{P}\left(z_{o}\right)$ represents one shot record and data matrix $\mathbf{P}\left(z_{0}\right)$ conveniently arranges the entire measurement volume (all involved shot records); matrices $\mathbf{D}\left(z_{0}\right)$ and $\mathbf{S}\left(z_{o}\right)$ represent the complete data acquisition information (geometry, field arrays and signatures); matrices $\mathbf{W}\left(z_{o}, z_{m}\right)$ and $\mathbf{W}\left(z_{m}, z_{o}\right)$ represent the forward propagation information between the surface $z_{o}$ and depth level $z_{m}$ (all involved upward and downward propagation operators) and matrix $\mathbf{R}^{+}\left(z_{m}\right)$ represents the reflectivity information (all involved reflection operators) at depth level $z_{m}$. Note that equation (1d) has been formulated for an acquisition geometry with a fixed detector geometry, i.e. detector matrix $\mathbf{D}\left(z_{o}\right)$ is the same for all source positions.

In the early nineties focusing has been introduced as a special version of target-oriented wavefield synthesis (Berkhout, 1992). Using this concept for upgoing as well as downgoing wavefields, the seismic imaging equations can be reformulated in terms of two consecutive numerical focusing processes (Berkhout, 1997; Thorbecke, 1997). 


\section{Migration of CFP gathers}

Let us consider the expression of a CFP gather due to focusing in emission

$$
\vec{P}_{J}\left(z_{o}, z_{f}\right)=\mathbf{P}\left(z_{o}\right) \vec{F}_{j}\left(z_{o}, z_{f}\right)
$$

with

$$
\mathbf{W}\left(z_{f}, z_{o}\right) \mathbf{S}\left(z_{o}\right) \vec{F}_{j}\left(z_{o}, z_{f}\right) \approx \vec{I}_{j}\left(z_{f}\right)
$$

where $\vec{F}_{j}\left(z_{o}, z_{f}\right)$ represents the focusing operator for focus point $\left(\vec{x}_{j}, z_{f}\right)$.

Equation (2a) formulates a weighted addition of shot records around lateral position $\vec{x}_{j}$. As was explained in Berkhout (1997), focusing in emission can be seen as the synthesis of the area1 shot record, $\vec{P}_{j}\left(z_{o}, z_{m}\right)$, due to target-oriented area1 source (Figure $1 \mathrm{~b}$ ):

$$
\vec{S}_{j}\left(z_{o}, z_{m}\right)=\mathbf{S}\left(z_{o}\right) \vec{F}_{j}\left(z_{o}, z_{m}\right)
$$

This means that a CFP gather can be migrated with a shot record migration algorithm. If we make use of the recursive $(\mathrm{x}, \mathrm{y}, \omega)$ algorithm, the computational scheme can be summarized as follows:

1. Forward extrapolate the focused source

$$
\vec{S}_{j}\left(z_{m}, z_{f}\right)=\mathbf{W}\left(z_{m}, z_{m-1}\right) \vec{S}_{j}\left(z_{m-1}, z_{f}\right)
$$

for $\mathrm{m}=1,2, \ldots \ldots, \mathrm{M}$.

2. Inverse extrapolate the CFP gather

$$
\vec{P}_{j}\left(z_{m}, z_{f}\right)=\mathbf{F}\left(z_{m}, z_{m-1}\right) \vec{P}_{j}\left(z_{m-1}, z_{f}\right)
$$

for $\mathrm{m}=1,2, \ldots, \mathrm{M}$.

3. Apply the scalar imagingprinciple

$$
\sum_{\omega}\left[\vec{P}_{j}\left(z_{m}, z_{f}\right)-\mathbf{R}^{+}\left(z_{m}\right) \vec{S}_{j}\left(z_{m}, z_{f}\right)\right]=\text { minimum }
$$

$\mathbf{R}^{+}\left(z_{m}\right)$ being a diagonal matrix of reflection coefficients.

The proposed migration scheme for 3-D multi-source CFP gathers may have significant advantages compared to the traditional migration scheme for 3-D single-source shot records, from the quality as well as the economy point of view. Experiments indicate that irregular sampling is not a problem, stabilization of the imaging principle (3c) is not critical, the spatial sampling of the focus point positions $\left(\vec{x}_{j}, z_{f}\right)$ may be sparse and we may choose $z_{f}=z_{f}(x, y)$.

It is also important to realize that the CFP image gathers may have a significantly better quality than the traditional image gathers (e.g. based on common-offset input), since many Fresnel zones contribute to one trace in the CFP image gather. In addition, updating the velocity model does not require the generation of new CFP gathers. Figure 2 shows a comparison between shot record migration (left) and CFP gather migration (right). Note the excellent quality of CFP-gather migration.

Figure 3 shows two image gathers based on shot records (left) and based on CFP gathers (right). Note the significantly better quality of the image gather based on CFP gathers.

\section{Conclusions}

To assess the validity of velocity models, it is recommended to analyse image gathers that are based on migrated CFP gathers. In the situation of misalignment, the operators should be updated by applying 'the principle of equal traveltime' (Berkhout, 1997). 


\section{Remark}

The focusing operators for the generation of 3-D multi-source CFP gathers may include the propagation properties of a complex near surface; this new approach to the 'statics problem' can be realized by making use of the principle of equal traveltime. This aspect makes the migration of CFP gathers also very promising for land data.

\section{References}

Berkhout, A.J., 1984, Seismic migration, practical aspects, vol. 14B, Elsevier, Amsterdam/North Holland.

Berkhout, A.J., 1992, Area1 shot record technology: J. Seis. Expl., 1, no. 2, 251-264.

Berkhout, A.J., 1997, Pushing the limits of seismic imaging, Part I and II, Geophysics, vol.62, no. 3 (May-June), p, 937 - 969.

Thorbecke, J., 1997, Common Focus Point Technology, PhD thesis, Delft.

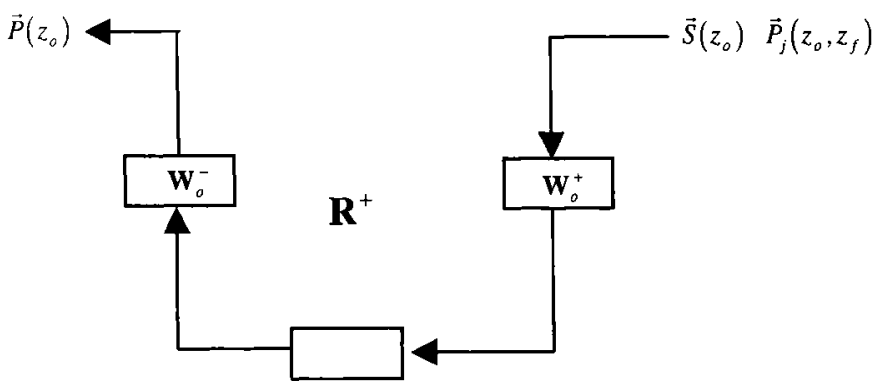

a. shot record

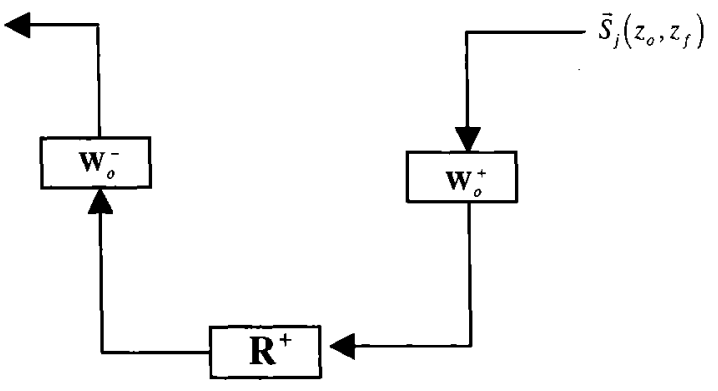

b. CFP gather

Figure 1: The forward model of a shot record (Ieft) and a CFP gather (right).

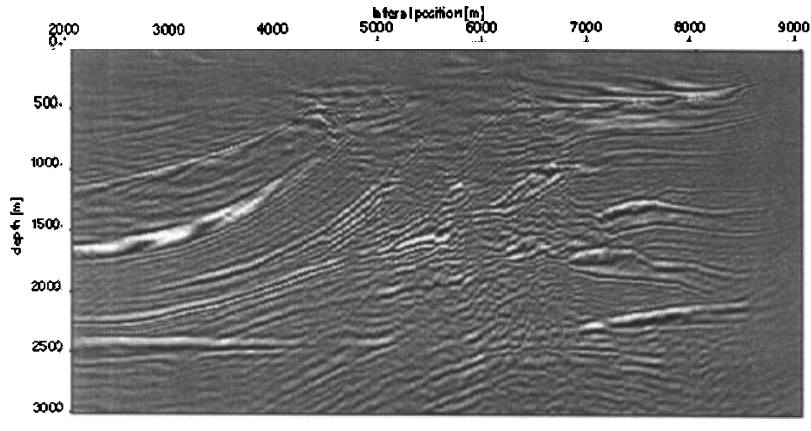

a. shot record migration

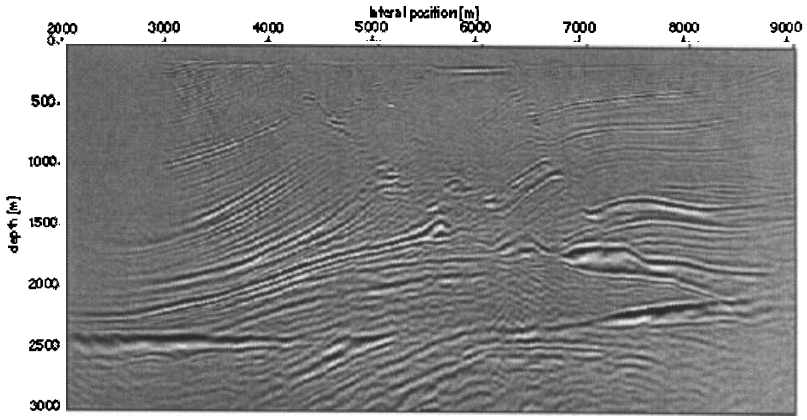

b. CFP gather migration

Figure 2: A comparison between migration of shot records (a) and CFP gathers (b), using a $500 \mathrm{~m}$ spacing between the source points and focus points respectively. 


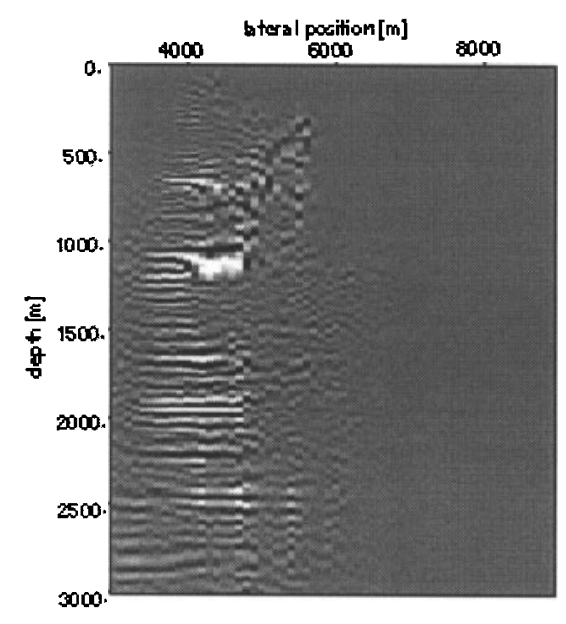

a. image gather based on shot record migration $(4000 \mathrm{~m})$

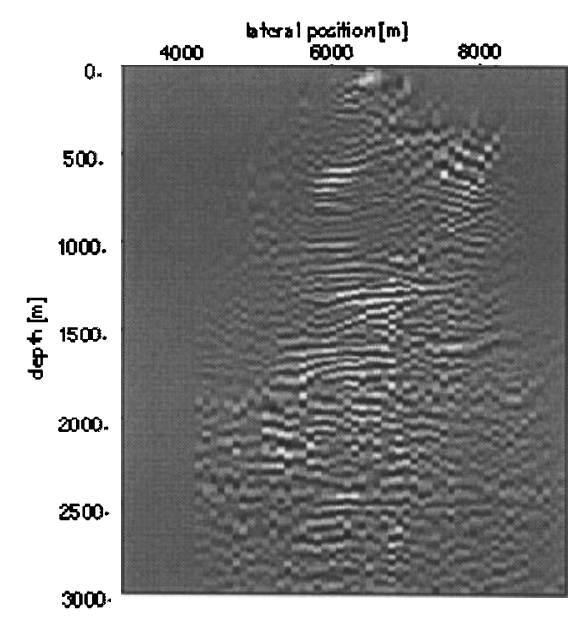

c. image gather based on shot record migration $(6000 \mathrm{~m})$

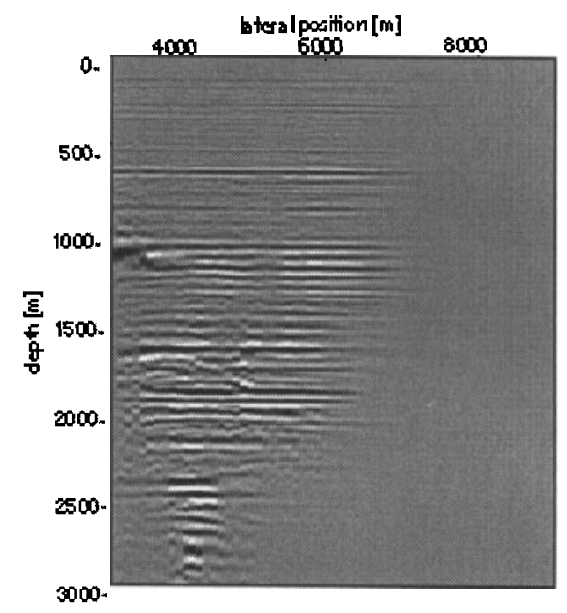

b. image gather based on CFP gather migration $(4000 \mathrm{~m})$

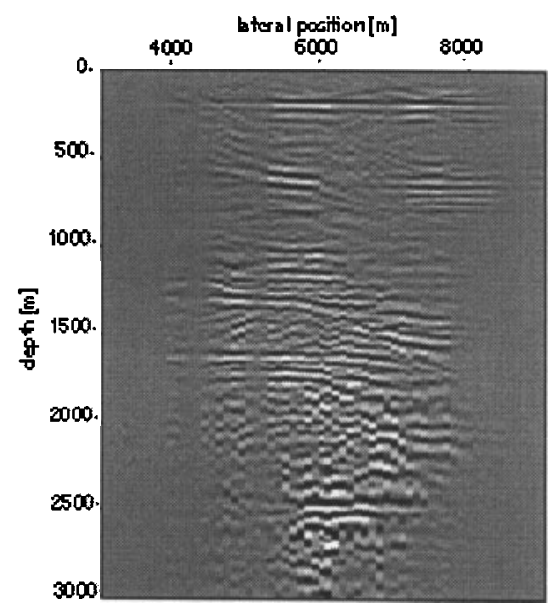

d. image gather based on CFP gather migration $(6000 \mathrm{~m})$

Figure 3: A comparison between image gathers based on shot record migration $(a, c)$ and CFP gather migration $(b, d)$ respectively. 